\title{
Primary diffuse leptomeningeal gliomatosis simulating tuberculous meningitis
}

\author{
J H Rees, N Balakas, A Agathonikou, S F Hain, G Giovanonni, C P Panayiotopoulos, \\ M Luxsuwong, $\mathrm{T}$ Revesz
}

University

Department of Clinical Neurology, Institute of Neurology, Queen

Square, London

WC1N 3BG, UK

J H Rees

G Giovanonni

Department of

Neuropathology

M Luxsuwong

T Revesz

Department of

Neurology,

Evangelismos

Hospital, Athens,

Greece

N Balakas

Department of Clinical Neurophysiology and

Epilepsies, St Thomas'

Hospital, London, UK

A Agathonikou

C P Panayiotopoulos

The Clinical PET

Centre

$S$ F Hain

Correspondence to:

Dr Jeremy Rees

j.rees@ion.ucl.ac.uk

Received 2 June 2000 and in revised form

4 August 2000

Accepted 16 August 2000

\begin{abstract}
Three patients are reported on who presented with communicating hydrocephalus due to presumed tuberculous meningitis. Subsequent clinical deterioration despite antituberculous chemotherapy prompted reassessment with FDG-PET scanning and meningeal biopsy in one case and repeat CSF cytology with special staining in the second. The third patient died and postmortem confirmed a diagnosis of primary diffuse leptomeningeal gliomatosis. In the first two patients, MRI of the entire neuraxis showed no evidence of a primary intraparenchymal tumour. These cases emphasise the need for repeated reassessment in patients with culture negative lymphocytic meningitis. In addition, this is the first report of FDG-PET scanning in leptomeningeal gliomatosis.

(F Neurol Neurosurg Psychiatry 2001;70:120-122)
\end{abstract}

Keywords: leptomeningeal; gliomatosis; tuberculous meningitis

The presenting features of tuberculous meningitis (TBM) consist of subacute headache, drowsiness, confusion, and meningism. Cerebrospinal fluid examination typically shows a raised protein concentration, a depressed glucose concentration, and a pleocytosis, initially polymorphs then lymphocytes. Failure to respond to treatment should prompt a search for fungal infections or malignancy.

Primary diffuse leptomeningeal gliomatosis (PDLG) is a rare condition whereby a glioma arises from heterotopic cell nests in the leptomeninges and produces a clinical picture similar to chronic infectious meningitis. This disease should not be confused with the much more often occurring secondary meningeal gliomatosis, due to primary CNS tumours, particularly medulloblastoma and glioblastoma, spreading along CSF pathways.

Cases of PDLG are usually only diagnosed at postmortem when a full microscopic examination of the CNS can be carried out to exclude a small primary tumour. We describe three cases of primary diffuse leptomeningeal gliomatosis, presenting as TBM, two of which were diagnosed in life and the third at postmortem.

\section{Case 1}

A 34 year old Greek woman was referred for further investigation of possible TBM, not responding to therapy. She presented with a progressive 2 month history of headaches, diplopia, and unsteadiness, rendering her bedbound. Neurological examination disclosed bilateral papilloedema, sixth nerve palsies, and neck stiffness. She was afebrile. Brain CT showed communicating hydrocephalus. Examination of CSF disclosed a raised opening pressure of $70 \mathrm{~cm}$ CSF, a lymphocyte pleocytosis $\left(17 / \mathrm{mm}^{3}\right)$, an increased CSF protein concentration $(0.69 \mathrm{~g} / \mathrm{dl})$, and a low $\mathrm{CSF} /$ plasma glucose ratio $(20 \%)$. A Mantoux test and seven CSF examinations including microscopy, culture, and PCR analysis were negative for mycobacteria. Cytology for malignant cells was also consistently negative. She was started on quadruple antituberculous therapy and dexamathasone. One month later, she went blind despite insertion of a ventriculoperitoneal shunt. Soon after, she became febrile with swinging temperatures of up to $38^{\circ} \mathrm{C}$ and developed increasing weakness of her legs due to a steroid myopathy. The dexamethasone was tailed off and she continued to deteriorate despite the addition of intramuscular streptomycin and intravenous isoniazid. Brain and spinal cord MRI with gadolinium showed patchy but widespread pial enhancement. Extensive investigations for a primary malignancy and for unusual infections were negative. Half body FDG-PET demonstrated intense uptake along the whole spinal cord extending into the medulla (figure). As there were no other areas of high uptake in the body she had a lumbar meningeal biopsy which showed a nerve root infiltrated by a glioma. As none of the scans had shown the presence of a primary tumour, a diagnosis of primary diffuse leptomeningeal gliomatosis was made. She died 5 weeks later in Greece. No postmortem was carried out.

\section{Case 2}

A 28 year old Asian man presented with a 3 month history of severe headache and backache associated with weight loss and night 


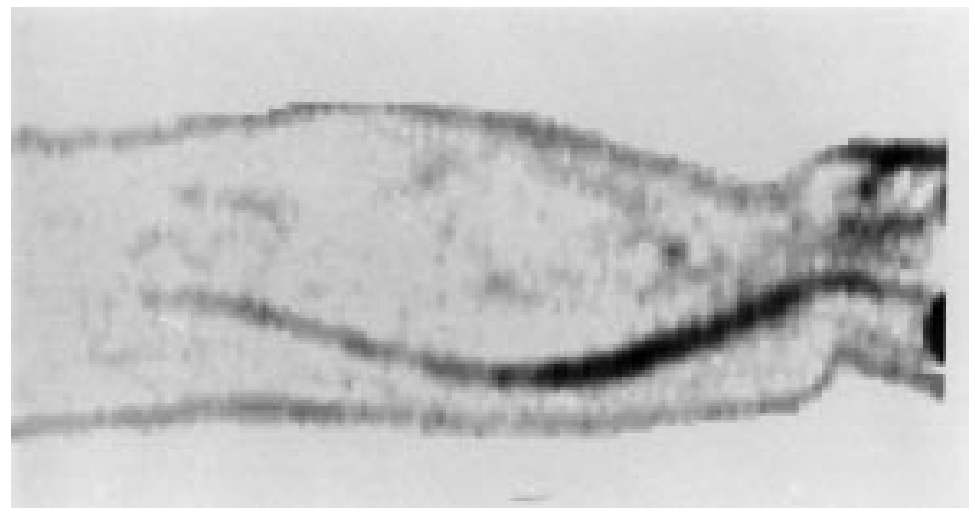

Figure 1 Case 1: Half body FDG-PET showing intense uptake along the length of the spinal cord extending up into the medulla. No other abnormalities are seen.

sweats. On admission, he was drowsy, cachectic, and in pain. There was marked neck stiffness and fundoscopy disclosed bilateral papilloedema. General examination was unremarkable. Brain CT showed communicating hydrocephalus with periventricular hypodensity. On MRI, there was marked thickening of the basal meninges with prominent gadolinium enhancement. No intra-axial lesions were visible on imaging of the whole neuraxis.

On lumbar puncture the opening pressure was $44 \mathrm{~cm}$ CSF. The CSF was pale yellow and contained 16 lymphocytes $/ \mathrm{mm}^{3}$. The CSF protein was $3.32 \mathrm{~g} / 1$ and the $\mathrm{CSF} /$ plasma glucose ratio was $20 \%$. Bacterial, fungal, and mycobacterial cultures and a PCR assay for $M$ tuberculosis were negative as was cytology for malignant cells. Chest radiography, CSF, and serum ACE concentrations were normal.

A presumptive diagnosis of TBM was made and he was started on quadruple chemotherapy. Over the next week his clinical condition deteriorated and he was started on dexamethasone. Repeat CSF cytology showed scattered large pleomorphic polygonal malignant cells which stained positive for glial fibrillary acidic protein (GFAP). A diagnosis of primary diffuse leptomeningeal gliomatosis was made on the basis of the imaging and the CSF cytology. He died 5 weeks after admission. His family declined a postmortem.

\section{Case 3}

A 51 year old man presented with a 6 week history of increasingly severe back and neck pain followed by generalised headache, nausea, and vomiting. On examination his head was fully extended and he had signs of severe meningism and early papilloedema. Brain CT showed communicating hydrocephalus and conspicuous contrast enhancement around the basal cisterns. A ventriculoperitoneal shunt and Ommaya reservoir were inserted. Ventricular CSF was turbid and had 20 cells $/ \mathrm{mm}^{3}$, mainly lymphocytes. The protein and glucose concentrations were normal. A gram and Ziehl-Neelsen stain were negative and culture showed no growth at 48 hours. Three further CSFs disclosed yellow fluid with variable pleocytosis (white cell count 60, 5, $\left.8 / \mathrm{mm}^{3}\right)$, raised protein concentations $(2.4,7.2$, and $4.1 \mathrm{~g} / \mathrm{l})$ and low CSF glucose concentrations $(1.7,0.8$, and $0.5 \mathrm{mmol} / \mathrm{l})$. All bacterial and TB cultures were negative, as were multiple cytology examinations. Brain and spinal cord MRI showed no intra-axial lesions and no pathological enhancement within the cervical cord. The thoracic cord was tethered posteriorly.

He was started on quadruple chemotherapy and dexamethasone, which improved the degree of neck retraction. After this, the CSF was reviewed by an experienced cytologist who noted the presence of an occasional atypical cell. On this basis, the antituberculous therapy was stopped and he was started on intrathecal methotrexate and systemic chemotherapy (cyclophosphamide, adriamycin, 5-FU) but died 2 days later.

At postmortem, the leptomeninges were thick and opaque, primarily over the base of the brain and around the spinal cord. This was due to the presence of a tumour affecting the basal meninges of the brain, including around the brainstem and present throughout the spinal meninges. This tumour was located exclusively in the leptomeninges and the morphological appearances and immunohistochemical findings were suggestive of an anaplastic astrocytoma (WHO grade III). No evidence of an intra-axial tumour mass was found after careful examination of the brain and spinal cord.

\section{Discussion}

These patients were initially thought to have TBM because of the characteristic clinical and CSF profile, but failure of response to treatment led to reconsideration of the diagnosis. All three cases reflect the considerable diagnostic difficulty in identifying the cause of a chronic meningitis with a persistently low CSF glucose when standard microbiological and cytological investigations and PCR analysis are negative.

To the best of our knowledge, case 1 is the first report of leptomeningeal gliomatosis in which PET has been used to search for an underlying systemic malignancy. The place of FDG-PET in differentiating benign from malignant disease is well established but certain infections including TB show FDG uptake equivalent to that of tumours in whole body studies. ${ }^{1}$ However, the presence of such a striking scan despite 5 months of chemotherapy and previous corticosteroids was thought to be more suggestive of a malignant process than an infective one.

The diagnosis of primary diffuse leptomeningeal gliomatosis should only be applied when no intraparenchymal tumour is found. ${ }^{2}$ Whereas we would agree that without a careful necropsy it is impossible to determine whether any gliomas are truly primary in the leptomeninges, a clinical antemortem diagnosis can be made when gadolinium enhanced MRI of the brain and spinal cord fails to demonstrate a primary tumour. The usefulness of CSF cytology is controversial. Previous reports suggest that patients with secondary meningeal gliomatosis often have tumour cells in their $\mathrm{CSF}^{3}$ but 
this has been refuted in a subsequent series of three patients, one of whom had PDLG ${ }^{4}$ and a case report. ${ }^{5}$ Cytology, however, is also helpful in excluding the more frequent conditions of carcinomatous and melanomatous meningitis.

1 O'Doherty MJ, Barrington SF, Campbell M, et al. PET patient. F Nucl Med 1997;38:1575-83.
2 Tumours of neuroglia and choroid plexus epithelium. In: Burger PC, Scheithauer BW, eds atlas of tumour pathology. Tumours of the central nervous system. Washington DC: Armed Forces Institute of Pathology; 1994:74-7.

3 Yung WA, Horten BC, Shapiro WR. Meningeal gliomatosis: a review of 12 cases. Ann Neurol 1980;8:605-8.

4 Whelan HT, Sung JH, Mastri AR. Diffuse leptomeningeal gliomatosis: report of three cases. Clin Neuropathol 1987;6: 164-8.

5 Wechsler LR, Gross RA, Miller DC. Meningeal gliomatosis with negative CSF cytology: the value of GFAP staining. Neurology 1984;34:1611-15. 